\title{
Estudio de Propiedades Físicas y Viscoelásticas de Panes Elaborados con Mezclas de Harinas de Trigo y de Arroz Integral
}

\author{
Armando Alvis*, Luis J. Pérez y Guillermo S. Arrazola \\ Universidad de Córdoba, Programa de Ingeniería de Alimentos, Fac. de Ciencias Agrícolas, \\ Grupo de Investigación Procesos y Agroindustria de vegetales, Carrera 6 No 76-103, Km 3, \\ vía Cereté, Córdoba-Colombia (e-mail: aalvis2@hotmail.com). \\ ${ }^{*}$ Autor a quien debe ser dirigida la correspondencia.
}

Recibido Nov. 22, 2010; Aceptado Dic. 28, 2010; Versión Final recibida Ene. 26, 2011

\begin{abstract}
Resumen
Se desarrollaron fórmulas de panes, empleando proporciones de harina de trigo comercial marca "Tres Castillos" (50 a 90\%) y harina de arroz integral comercial marca "Diana" (10 a 40\%) y grasa entre 0 y $6 \%$. Se estudian las propiedades físicas y viscoelásticas a través de técnicas de medición simples para parámetros físicos, un análisis de perfil de textura y la prueba de relajación de esfuerzos. De los resultados se apreció que el volumen específico es el parámetro físico más representativo para los panes. Las propiedades de textura resultaron significativas para $p<0.05$ y oscilaron para dureza entre 4.0 a $10.7 \mathrm{~N}$ y elasticidad entre 9 a $12 \mathrm{~mm}$ y el ajuste del modelo de Peleg permitió corroborar la naturaleza viscoelástica del pan. Se concluyó, que se pueden obtener fórmulas de panes de harina de trigo con harina de arroz integral que presenten propiedades físicas y viscoelásticas aceptables y de buena calidad.
\end{abstract}

Palabras claves: pan, arroz integral, dureza, elasticidad, volumen específico

\section{Study of Viscoelastic and Physical Properties of Breads Made with mixed Wheat and Brown Rice Flours}

\begin{abstract}
New formulas for breads, using different proportions of commercial wheat flour of the brand "Tres Castillos" (50 a 90\%) and brown rice flour trade mark of the brand "Diana" (10 a 40\%), and fat from 0 to $6 \%$, have been formulated. Physical and viscoelastic properties are determined using simple techniques for measuring physical parameters and texture profile analysis and stress relaxation test for viscoelastic properties. From the results it was found that the specific volume is the most representative physical parameter for breads. The texture properties were highly significant at $p$ $<0.05$ and for hardness ranged from 4.0 to $10.7 \mathrm{~N}$ and elasticity from 9 to $12 \mathrm{~mm}$. The fitting to the Peleg model corroborated the viscoelastic nature of the breads. It was concluded that formulas for combining bread wheat flour with rice flour having acceptable physical and viscoelastic properties and of good quality can be obtained.
\end{abstract}

Keywords: bread, brown rice, hardness, elasticity, specific volume 


\section{INTRODUCCIÓN}

En Colombia; se ha incrementado en los últimos tiempos la importación del trigo para compensar el desbalance que ha generado su alta demanda y la poca producción de este importante grano para abastecer las necesidades internas de producción de harina y su posterior uso en panificación. Se han buscado alternativas de implementación de otros tipos de harinas para la industria panadera; de hecho se han realizado investigaciones para ayudar a resolver esta situación en donde se sustituye parcialmente la harina de trigo por las de yuca, arroz, maíz, sorgo y otros, y se ha demostrado que desde el punto de vista tecnológico, se puede producir pan con estas mezclas de harinas y obtener características comparables a las del pan con $100 \%$ harina de trigo (Henao y Aristizábal, 2009).

El pan es uno de los productos alimenticios a base de cereales que más se consume en el mundo, sin embargo, debido a que generalmente es elaborado con harina de trigo, no es consumible por toda la población, ya que existen personas que presentan intolerancia a las prolaminas, proteínas mayoritarias del trigo (también de la avena, la cebada y el centeno). Pese a las propiedades benéficas que ofrece el pan elaborado con agregado de harina de arroz integral tanto por el valor nutritivo que representa como por su escaso contenido de prolaminas, incursionar en la producción y comercialización de éste representa un gran reto, ya que raramente cuenta con buena aceptabilidad por parte de los consumidores (Sánchez et al., 2008), debido a que sus características físicas y sensoriales difieren de panes originales hechos con harina de trigo, por cuanto su masa posee pocas proteínas formadoras de gluten que son imprescindibles para obtener panes de excelentes propiedades y características Físicas (Clerici y El-Dash, 2006; Pedrosa et al., 2009)

Marco y Molina (2008); Sánchez et al., (2008) y Pedrosa et al., (2009), han empleado el arroz y otros cereales o tubérculos como objeto de estudio para sustituir totalmente al trigo en la fórmula del pan como producto para celíacos, ya que su harina se caracteriza por poseer propiedades hipoalergénicas, un sabor suave, bajo contenido en prolaminas, poco contenido en grasas y baja proporción de sodio, lo cual la hacen fácilmente digerible (Gujral y Molina, 2004; Marco y Molina, 2008). Adicionalmente, el arroz integral contribuye en las propiedades nutricionales del pan, ya que contiene cantidades considerablemente más elevadas de proteínas y minerales que el arroz blanco (Itani et al., 2002; Lamberts et al., 2007; Renzetti y Arendt, 2009). Por su parte Veluppillai et al. (2010) utilizaron la harina de arroz malteado para sustituir parcialmente la harina de trigo en la fórmula del pan. Por lo tanto el desarrollo de productos horneados empleando harina de arroz integral es una alternativa para mejorar su valor nutricional, reducir el uso del trigo y por ende importaciones de este grano. Además la harina puede aprovecharse como subproducto del arroz, ya que generalmente es obtenida a partir de los granos rotos durante el proceso de molienda.

El estudio y conocimiento de la textura como propiedad física del alimento es de suma importancia para determinar la aceptabilidad del mismo, el consumidor prefiere aquellos alimentos que resulten agradables sensorialmente, esta aceptación es determinante en alimentos como carne y sus derivados así como en los productos crujientes entre otros, también es importante en alimentos en los que la textura, a pesar de no ser una característica dominante, contribuye a su calidad global; algunos ejemplos se representan en frutas, hortalizas y pan, entre otros (Ibarz et al., 2000). Para caracterizar los productos obtenidos con las mezclas de harina de trigo y arroz integral, es de gran utilidad estudiar sus propiedades mecánicas y viscoelásticas ya que sus resultados pueden ser útiles para obtener información fundamental acerca de los aspectos básicos de la textura y su relación con la estructura y con atributos sensoriales (Steffe, 1996).

Un material viscoelástico tiene diversas constantes reológicas; es decir, no hay una constante simple para materiales viscoelásticos tal como el módulo elástico, ya que este cambia con el tiempo. Las constantes reológicas para este material se representan por una ecuación que expresa el módulo en función del tiempo, así que si un material viscoelástico se somete a la acción de un esfuerzo constante, la manera en la cual se deforma cambia con el tiempo, una de las pruebas más utilizadas para el análisis de los alimentos es la curva de relajación de esfuerzos, que se describe como la habilidad de un material para disipar un esfuerzo impuesto bajo 
condiciones de deformación constante (Mohan y Quintero, 2005). Las deformaciones deben ser muy pequeñas (menos del $3.0 \%$ ) para asegurar que el material se encuentre en la región elástica y pueda utilizarse la modelación (Skinner, 1983). Sin embargo, en la literatura revisada pueden encontrarse trabajos que utilizan deformaciones mucho más elevadas (5, 10 y $20 \%$ ) (Mohan y Quintero, 2005). Como objetivo del estudio se plantea desarrollar fórmulas de panes de trigo con incorporación parcial de harina de arroz integral para obtener resultados de las mediciones de algunas de sus propiedades físicas y viscoelásticas y determinar su calidad.

\section{MATERIALES Y MÉTODOS}

Para la evaluación de las propiedades físicas y viscoelásticas de los panes elaborados con agregado de harina de arroz, se utilizaron panes de molde siguiendo el orden aleatorio arrojado por la matriz experimental mostrado en la tabla 1. En éstas, se variaron las proporciones de harina de trigo, harina de arroz integral y grasa y se mantuvieron constantes el resto de los ingredientes (azúcar, sal, agua, levadura y mejorador). En la elaboración de los panes se emplearon harina de trigo comercial marca "3 Castillos", harina de arroz integral comercial marca "Diana", margarina (con índice máximo de peróxido 10 meq-g $\mathrm{O}_{2} / \mathrm{kg}$ grasa e índice máximo de acidez $0,6 \mathrm{mg} \mathrm{KOH} / \mathrm{kg}$ grasa) marca "La fina", Levadura y mejorador (compuesto por $\alpha$-amilasa, ácido ascórbico y harina de trigo como diluyente) marca "Levapan", sal, azúcar refinada y agua potable.

Las propiedades físicas más relevantes medidas a los panes fueron: peso (g); el cual se midió en una balanza técnica de precisión $\pm 0.01 \mathrm{~g}$, altura $(\mathrm{cm})$; que se realizó empleando un pie de rey de precisión $\pm 0.02 \mathrm{~mm}$, volumen $\left(\mathrm{cm}^{3}\right)$, por desplazamiento de semillas utilizando un volumenómetro y volumen específico $\left(\mathrm{cm}^{3} / \mathrm{g}\right)$; a través de la relación volumen/peso. La textura y las propiedades viscoelásticas se realizaron con un texturómetro TA-HDplus de la firma Stable Micro Systems, utilizando como celda de prueba un plato de compresión de $35 \mathrm{~mm}$ de diámetro y el cálculo de los parámetros evaluados se llevó a cabo a través del software TEE 32. En todos los casos, el pan se cortó en forma de rebanada de $25 \mathrm{~mm}$ de espesor y los ensayos se realizaron a la temperatura ambiente $\left(25^{\circ} \mathrm{C}\right)$. Las pruebas utilizadas fueron las siguientes:

Análisis de Perfil de Textura (TPA). Se aplicó doble compresión sucesiva a una rebanada de 25 $\mathrm{mm}$ de espesor hasta un $50 \%$ de su altura a la velocidad de $4 \mathrm{~mm} / \mathrm{s}$, con un tiempo de reposo entre ambas compresiones de $2 \mathrm{~s}$. A partir de los datos fuerza vs tiempo, se calculó la dureza, como la fuerza máxima en la primera compresión y la elasticidad, definida como la distancia que recupera el producto en la segunda compresión.

Prueba de relajación de esfuerzos. La rebanada se comprimió hasta el $20 \%$ de su altura a una velocidad de $0,5 \mathrm{~mm} / \mathrm{s}$ y se permitió su relajación por espacio de 5 minutos. Se ajustó el modelo de Peleg (1979) para calcular los parámetros a y b usnado los datos de fuerza vs tiempo.

Para determinar las fórmulas del pan con harina de arroz integral se utilizó un diseño estadístico de experimentos mixto, empleando un $D$ óptimo para la parte correspondiente a las mezclas, variando las proporciones de harina de trigo y arroz integral entre $(60$ - 90/10 - 40) y un diseño factorial para la parte correspondiente a la proporción de grasa en la fórmula que varió entre 0 6\% (Montgomery, 2002). La matriz del diseño experimental se muestra en la tabla 1.

Se elaboraron además tres réplicas de fórmulas $100 \%$ harina de trigo y $4 \%$ de grasa, manteniendo el resto de los ingredientes igual que en el diseño, para evaluar la influencia de la harina de arroz en los resultados de la panificación. A estas muestras se realizaron los mismos análisis físicos y viscoelásticos que al resto de los panes. Las variables respuestas fueron: Propiedades físicas: altura (h), volumen específico (VE) dureza (d) y elasticidad (e). Los resultados de las variables respuestas, se procesaron estadísticamente a través de la Metodología de Superficie de Respuesta, obteniendo así los modelos de regresión mediante los cuales fue posible explicar su comportamiento y la influencia que sobre ellas ejercen los componentes harina de trigo, harina de arroz y la grasa. Tanto para la obtención de la matriz del diseño experimental como para el análisis de los resultados obtenidos de las propiedades físicas medidas a los panes se utilizó el programa estadístico Design - Expert versión 6.0.1 (Stat-Ease Inc., 2000). 
Tabla 1. Corridas experimentales del diseño mixto.

\begin{tabular}{|c|c|c|c|c|c|c|c|}
\hline Corrida & $\begin{array}{c}\mathrm{C}_{1} \\
\text { Harina de } \\
\text { trigo }\end{array}$ & $\begin{array}{c}\mathrm{C}_{2} \\
\text { Harina de } \\
\text { arroz integral }\end{array}$ & $\begin{array}{c}\mathrm{X}_{1} \\
\text { Grasa }\end{array}$ & Corrida & $\begin{array}{c}\mathrm{C}_{1} \\
\text { Harina de } \\
\text { trigo }\end{array}$ & $\begin{array}{c}\mathrm{C}_{2} \\
\text { Harina de } \\
\text { arroz integral }\end{array}$ & $\begin{array}{c}\mathrm{X}_{1} \\
\text { Grasa }\end{array}$ \\
\hline 1 & 67.50 & 32.50 & 4.50 & 10 & 82.50 & 17.50 & 1.50 \\
\hline 2 & 60.00 & 40.00 & 6.00 & 11 & 75.00 & 25.00 & 6.00 \\
\hline 3 & 67.50 & 32.50 & 1.50 & 12 & 60.00 & 40.00 & 6.00 \\
\hline 4 & 90.00 & 10.00 & 3.00 & 13 & 60.00 & 40.00 & 0.00 \\
\hline 5 & 75.00 & 25.00 & 3.00 & 14 & 75.00 & 25.00 & 0.00 \\
\hline 6 & 90.00 & 10.00 & 6.00 & 15 & 60.00 & 40.00 & 0.00 \\
\hline 7 & 90.00 & 10.00 & 6.00 & 16 & 90.00 & 10.00 & 0.00 \\
\hline 8 & 90.00 & 10.00 & 0.00 & 17 & 82.50 & 17.50 & 4.50 \\
\hline 9 & 60.00 & 40.00 & 3.00 & & & &
\end{tabular}

\section{RESULTADOS Y DISCUSIÓN}

En la tabla 2 se muestran los resultados del volumen específico (VE) y la altura, en ella se aprecia que los valores de VE para las diferentes fórmulas desarrolladas se encontraron entre 3.61 y 5.75 $\mathrm{cm}^{3} / \mathrm{g}$. El valor máximo obtenido por el diseño se acercó al VE del pan $100 \%$ harina de trigo que fue de $5.8 \mathrm{~cm}^{3} / \mathrm{g}$, y se aproximaron al reportado por El-Dash et al. (1982) para panes blancos (6 $\mathrm{cm}^{3} / \mathrm{g}$ ). Por su parte la altura de los panes con harina de arroz integral osciló entre 9.1 a $13.3 \mathrm{~cm}$, también con su valor máximo muy cercano al del pan $100 \%$ harina de trigo $(13.5 \mathrm{~cm})$.

Tabla 2. Resultados de Altura y Volumen específico

\begin{tabular}{|c|c|c|c|c|c|}
\hline Corrida & $\begin{array}{c}\text { Altura } \\
(\mathrm{cm})\end{array}$ & $\begin{array}{c}\text { Volumen específico } \\
\left(\mathrm{cm}^{3} / \mathrm{g}\right)\end{array}$ & Corrida & $\begin{array}{c}\text { Altura } \\
(\mathrm{cm})\end{array}$ & $\begin{array}{c}\text { Volumen específico } \\
\left(\mathrm{cm}^{3} / \mathrm{g}\right)\end{array}$ \\
\hline 1 & 9.5 & 4.20 & 10 & 10.8 & 4.40 \\
\hline 2 & 10.0 & 3.71 & 11 & 10.5 & 4.36 \\
\hline 3 & 10.7 & 4.13 & 12 & 9.10 & 3.68 \\
\hline 4 & 13.3 & 4.41 & 13 & 9.10 & 3.69 \\
\hline 5 & 11.5 & 4.30 & 14 & 10.40 & 4.05 \\
\hline 6 & 12.0 & 5.71 & 15 & 9.40 & 3.61 \\
\hline 7 & 12.0 & 5.75 & 16 & 11.70 & 5.02 \\
\hline 8 & 12.3 & 4.84 & 17 & 11.00 & 4.50 \\
\hline 9 & 10.4 & 4.33 & & & \\
\cline { 1 - 3 }
\end{tabular}

El análisis de varianza de la regresión resultó significativo ( $p<0.0001)$, la ecuación explica el 99\% de las variaciones del VE y el modelo de mejor ajuste fue de tipo cuadrático. Pedrosa et al. (2009), obtuvieron una regresión cuadrática para el volumen específico de los panes de harina de arroz extrudida ácida, para la altura el análisis de varianza no fue significativo, por lo que no se halló un modelo de regresión que explicara su comportamiento. La ecuación de regresión obtenida se presenta a continuación:

$V E=4.43 C_{1}+4.32 C_{2}+0.39 C_{1} X_{1}+0.91 C_{1} X_{1}^{2}-0.65 C_{2} X_{1}^{2}-0.88 C_{1} C_{2} X_{1}^{2}$

La ecuación 1 muestra, que tanto la proporción de harina de trigo como la de harina de arroz tienen la mayor influencia positiva sobre el volumen específico, manteniéndose ambos coeficientes casi iguales $\left[R^{2}=0.995\right]$. Sin embargo, Gujral y Molina (2004), Marco y Molina (2008), y Pedrosa et al. (2009), emplearon mayores proporciones de harina de arroz y encontraron una disminución apreciable de este parámetro físico, por lo que la cantidad utilizada de este componente debe limitarse.En la ecuación 1 se observa, que existe una interacción positiva entre 
el contenido de grasa y la harina de trigo, pero negativa al interactuar con el arroz. Esto se atribuye a que la grasa al interactuar con la harina de trigo mejora el efecto lubricante y sella los poros de la red del gluten Lucas (2008); sin embargo, el arroz resulta una carga que no aporta gluten y al aumentar tanto la grasa como el arroz se diluye el gluten presente, afectando el volumen del pan.

Si se desea obtener altos valores de VE, debe emplearse la mayor cantidad de harina de trigo y la menor cantidad de harina de arroz posible, con un porcentaje de grasa intermedio (3\%) para no afectar mucho el VE, tal como se aprecia en la figura 1, que muestran los resultados, con los valores máximos y mínimos codificados para los componentes de la mezcla.

$$
\begin{aligned}
& \mathrm{VE}=4.32-0.65=3.67 \quad \text { Para } \mathrm{C}_{1}=0 \text { y } \mathrm{C}_{2}=1 \text { (Máximo de arroz) } \\
& \mathrm{VE}=4.43+0.39+0.91=5.73 \quad \text { Para }_{1}=1 \text { y } \mathrm{C}_{2}=0 \text { (Máximo de trigo) }
\end{aligned}
$$

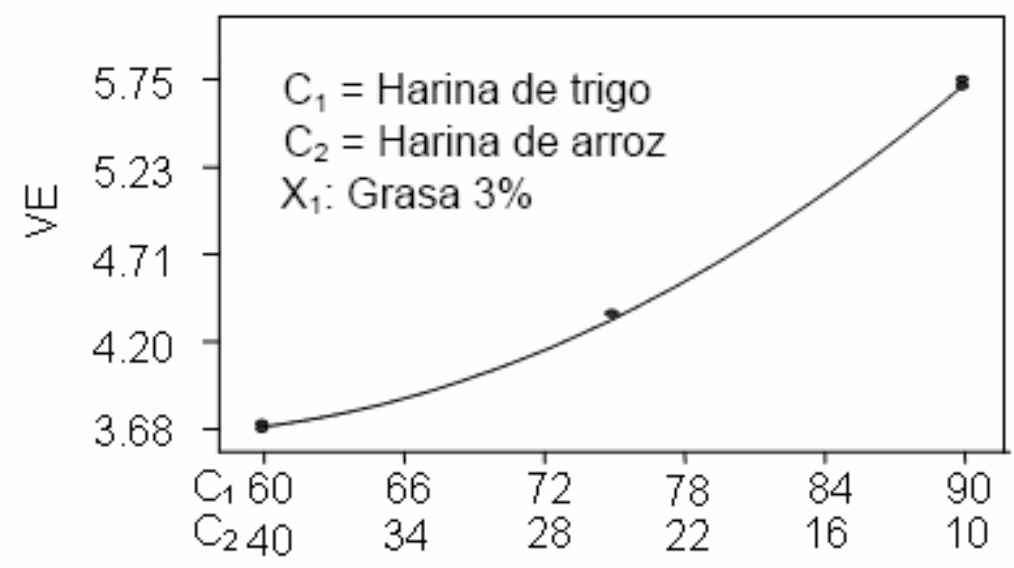

Fig. 1: Gráfico del modelo ajustado para el volumen específico

Los resultados de la elasticidad y dureza se presentan en la tabla 3. Los valores de dureza se encuentran en el intervalo de 4.0 a $11.0 \mathrm{~N}$. Machado (1996) ofrece valores de la dureza de pan de arroz calculada por el TPA después de $1 \mathrm{~h}$. de enfriamiento entre 14.79 y $104.79 \mathrm{~N}$, señalando que son panes muy duros. También Gujral y Molina (2004), muestran resultados alrededor de $14.0 \mathrm{~N}$ para panes elaborados con $100 \%$ harina de arroz, confirmando una firmeza superior a la del pan $100 \%$ de trigo, por lo que recomiendan el uso de enzimas tal como la alfa amilasa y la ciclodextrina glucoxiltransferasa (CGTasa) ya que producen un incremento considerable en el volumen específico y reducen la firmeza, además disminuyen la habilidad de retrogradación de la amilopectina del arroz durante el almacenamiento del pan.

En este estudio la dureza del pan $100 \%$ harina de trigo obtuvo el menor resultado de todos $(\mathrm{d}=$ $3.92 \mathrm{~N}$ ) este valor se encuentra cercano al de las fórmulas que poseen la mayor proporción de harina de trigo, la menor proporción de harina de arroz y el mayor contenido de grasa (Corridas 4 , $6,7)$.

La ecuación de regresión obtenida tuvo coeficientes significativos $(p<0.0001)$.

$d=5.32 C_{1}+10.06 C_{2}-0.92 C_{1} X_{1}$

De acuerdo con el valor del coeficiente de determinación $\left[R^{2}=0.93\right]$ puede decirse que el modelo es de tipo predictivo y explica el $93 \%$ de las variaciones, ecuación 4 . La prueba de falta de ajuste resultó no significativa, el análisis de los residuos estandarizados sigue una distribución normal y no se detectaron observaciones atípicas. 
Tabla 3. Resultados de Dureza y Elasticidad

\begin{tabular}{|c|c|c|c|c|c|}
\hline Corridas & $\begin{array}{l}\text { Dureza (d) } \\
\qquad(\mathrm{N})\end{array}$ & $\begin{array}{c}\text { Elasticidad }(e) \\
(\mathrm{mm})\end{array}$ & Corridas & $\begin{array}{c}\text { Dureza (d) } \\
(\mathrm{N})\end{array}$ & $\begin{array}{c}\text { Elasticidad (e) } \\
\qquad(\mathrm{mm})\end{array}$ \\
\hline 1 & 7.95 & 11.2 & 10 & 8.26 & 11.3 \\
\hline 2 & 9.64 & 10.2 & 11 & 6.05 & 10.9 \\
\hline 3 & 8.51 & 10.9 & 12 & 9.79 & 9.90 \\
\hline 4 & 4.92 & 11.3 & 13 & 10.7 & 9.00 \\
\hline 5 & 6.82 & 11.2 & 14 & 8.83 & 11.20 \\
\hline 6 & 4.50 & 11.8 & 15 & 10.58 & 9.20 \\
\hline 7 & 4.0 & 12.0 & 16 & 6.83 & 10.90 \\
\hline 8 & 5.32 & 10.9 & 17 & 6.88 & 10.70 \\
\hline 9 & 10.28 & 9.70 & & & \\
\hline
\end{tabular}

En ella el componente harina de arroz es el de mayor influencia de forma tal que su incremento provoca un aumento en la dureza. Por su parte la harina de trigo y la grasa en alta proporción (6\%) mejoran la textura, lográndose un pan más suave figura 2.

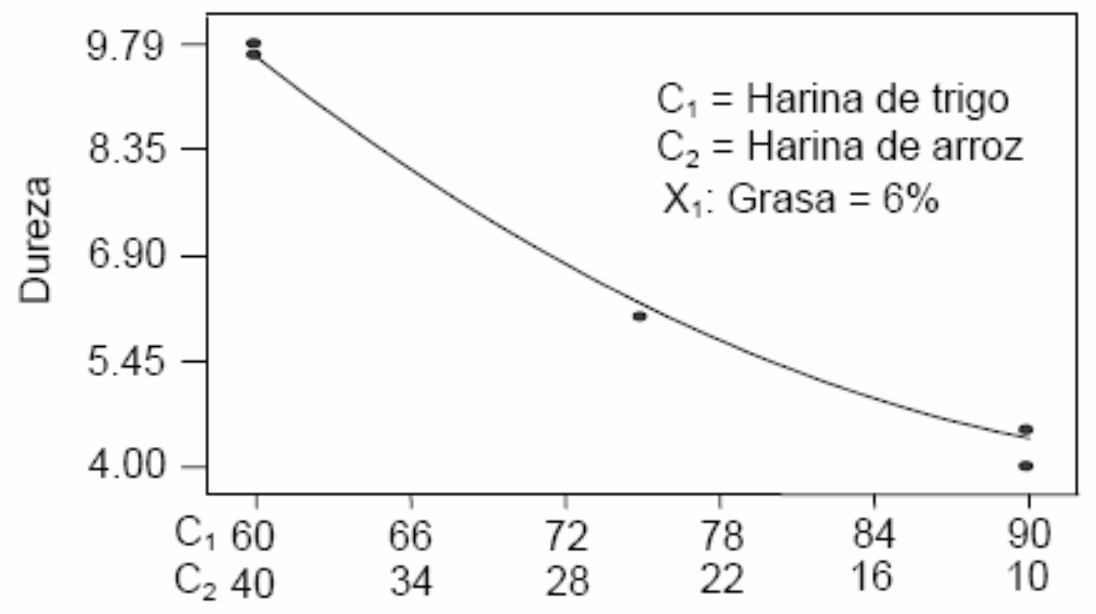

Fig. 2: Gráfico del modelo ajustado para la dureza

Para la elasticidad, la ecuación obtenida es la siguiente:

$\mathrm{e}=11.29 \mathrm{C}_{1}+9.7 \mathrm{C}_{2}+2.59 \mathrm{C}_{1} \mathrm{C}_{2}+0.47 \mathrm{C}_{1} \mathrm{X}_{1}+0.51 \mathrm{C}_{2} \mathrm{X}_{1}-2.71 \mathrm{C}_{1} \mathrm{C}_{2} \mathrm{X}_{1}$

Resultó significativa para $p<0.0001$. La prueba de falta de ajuste resultó no significativa $\left[R^{2}=0.90\right]$, el modelo ajustado explica el $90 \%$ de las variaciones de la elasticidad en el pan, el análisis de los residuos estandarizados siguen una distribución normal y no se detectaron observaciones atípicas.

Para la ecuación 5, la proporción de harina de trigo como la de arroz integral tiene el mayor efecto positivo de todas las variables consideradas. Este tipo de pan posee una elasticidad moderada y un incremento superior al $25 \%$ del componente C2, provoca finalmente una rápida disminución de la elasticidad, tal como se aprecia en la figura 3. En cuanto al contenido de grasa, los tres últimos términos del modelo parecen estar equilibrados, esto significa que un incremento en el contenido de grasa (dentro de los límites fijados por el experimento), induce en parte a un incremento de la elasticidad que se reduce cuando interviene el último término de la expresión. 


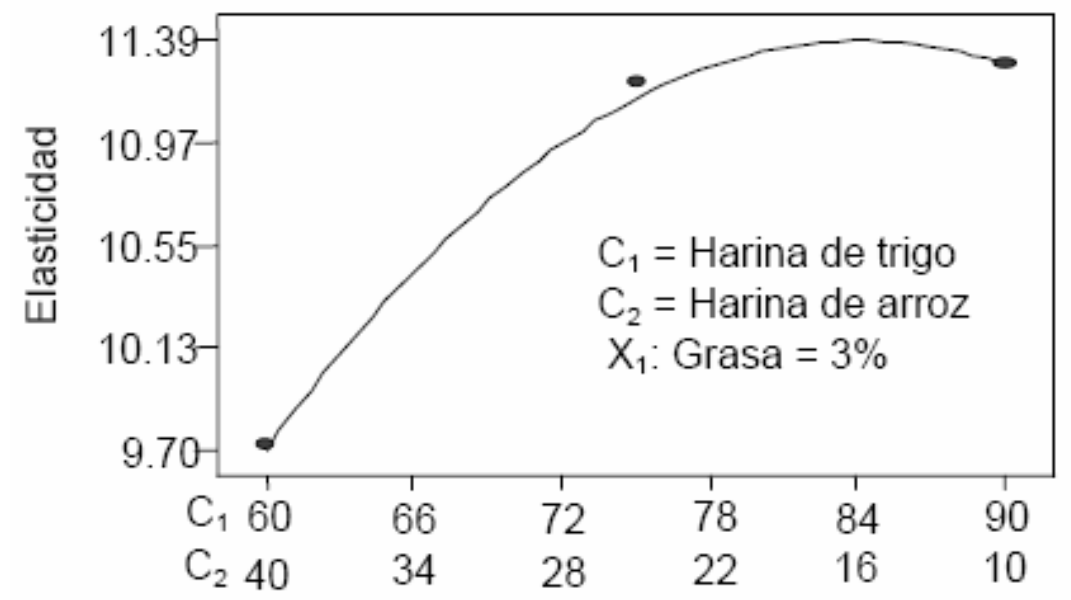

Fig. 3: Gráfico del modelo ajustado para la elasticidad.

En la figura 4 se observan valores más elevados de elasticidad cuando se emplea una alta proporción de harina de trigo y poca cantidad de harina de arroz integral, adicionando el máximo contenido de grasa (6\%).

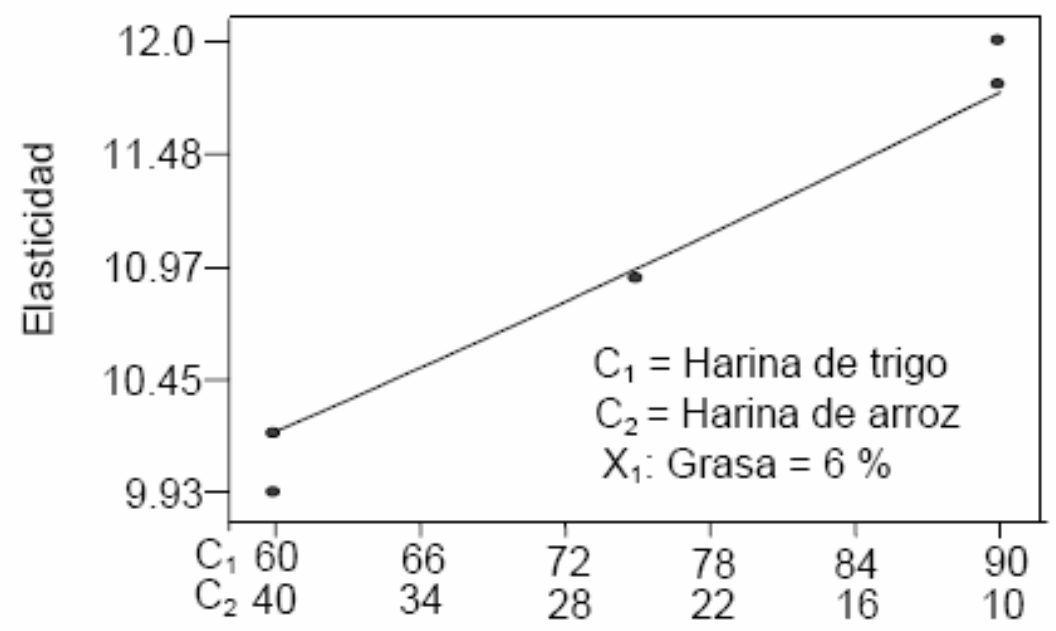

Fig. 4: Grafico del modelo ajustado para la elasticidad

En los alimentos viscoelásticos (pan), es frecuente que solo ocurra una recuperación parcial de su estructura cuando se somete a determinadas deformaciones, ya que la red tridimensional elástica se desintegra bajo el efecto de la tensión o compresión. Cuanto más completa y resistente sea esta red, más acusada será la elasticidad y cuanto más fácilmente se desintegre la red, mayor será el componente viscoso (Muller, 1973).

Los resultados del ajuste y el coeficiente de determinación del modelo de Peleg (1979) se muestran en la Tabla 4 y en ella se aprecia que los valores de "a" se encuentran en un intervalo de 0.51 a 0.71 y los valores de "b" entre 0.029 y 0.114 .

El parámetro "a" representa el valor asintótico al cual cae el esfuerzo durante la relajación, estos valores se encuentran $0<a<1$ lo cual corrobora la naturaleza viscoelástica del producto. Existe cierta tendencia a que los valores de "a" más elevados corresponden a las fórmulas con mayor contenido de harina de trigo (Corridas 4 y 16) y que además estos resultados son similares a los obtenidos para el pan elaborado con $100 \%$ harina de trigo $(a=0.72)$. En todos los casos "b" tiene valores muy cercanos a cero, lo cual indica que la velocidad de relajamiento es muy lenta y que el producto se aproxima al sólido elástico ideal. 
Tabla 4. Resultados de los parámetros "a" y "b" de Peleg

\begin{tabular}{|c|c|c|c|c|c|c|c|}
\hline \multirow[t]{2}{*}{ Corrida } & \multicolumn{3}{|c|}{ Constantes de Peleg } & \multirow[t]{2}{*}{ Corrida } & \multicolumn{3}{|c|}{ Constantes de Peleg } \\
\hline & A & $b$ & $\mathrm{R}^{2}$ & & a & $B$ & $\mathrm{R}^{2}$ \\
\hline 1 & 0.57 & 0.042 & 0.996 & 10 & 0.54 & 0.042 & 0.997 \\
\hline 2 & 0.61 & 0.114 & 0.991 & 11 & 0.58 & 0.039 & 0.996 \\
\hline 3 & 0.58 & 0.029 & 0.996 & 12 & 0.61 & 0.033 & 0.996 \\
\hline 4 & 0.71 & 0.043 & 0.980 & 13 & 0.64 & 0.036 & 0.996 \\
\hline 5 & 0.60 & 0.031 & 0,995 & 14 & 0.58 & 0.067 & 0.990 \\
\hline 6 & 0.69 & 0.066 & 0.984 & 15 & 0.63 & 0.058 & 0.997 \\
\hline 7 & 0.51 & 0.049 & 0.996 & 16 & 069 & 0.078 & 0.995 \\
\hline 8 & 0.63 & 0.053 & 0.988 & 17 & 0.56 & 0.038 & 0.997 \\
\hline 9 & 0.53 & 0.044 & 0.998 & & & & \\
\hline
\end{tabular}

\section{CONCLUSIONES}

El volumen específico es el parámetro físico más representativo para evaluar los panes con agregado de harina de arroz integral, encontrándose en valores entre 3.61 y $5.75 \mathrm{~cm}^{3} / \mathrm{g}$ y ajustándose a una ecuación de regresión altamente significativa $p<0.0001$, por su parte los resultados de dureza y elasticidad reportados para el pan y que oscilaron entre $4,0-10.7 \mathrm{~N}$ y $9-$ $12 \mathrm{~mm}$ respectivamente, fueron altamente significativos $(p<0,05)$ e influyeron en las características físicas y de calidad del pan.

Los parámetros a y b del modelo de Peleg, reportados en un intervalo entre 0,51 a 0,71 y 0,029 a 0,114 respectivamente, corroboran la naturaleza viscoelástica del pan elaborado con agregado harina de arroz integral. Es posible la obtención de panes de calidad aceptable con una proporción HT/HA/G (80:20:3.7) que presente buenas propiedades físicas y viscoelásticas.

\section{AGRADECIMIENTOS}

Los autores expresan su total agradecimiento a la Universidad de Córdoba, al grupo de Investigación Procesos y Agroindustria de Vegetales, al Codecyt y al Instituto de Investigaciones para la Industria Alimentaria (IIIA).

\section{REFERENCIAS}

Clerici, M. y A. El-Dash; Farinha extrusada de arroz como substituto de glúten na produção de pão de arroz, Archivos Latinoamericanos de Nutrición 56(2): 288-294 (2006).

El-Dash, A., C. Camargo y N. Díaz; Fundamentos de Tecnología de Panificação, Série AgroIndustrial, 400 (1982).

Gujral, H. y C. Molina; Improvement of the bread making quality of rice flour by glucose. Food Research International. 37(1): 75-81 (2004).

Henao S. y J. Aristizabal, Influencia de la variedad de yuca y nivel de sustitución de harinas compuestas sobre el comportamiento reológico en panificación, Revista Ingeniería e Investigación 29 (1): 39-46 (2009).

Ibarz, A., G. Barbosa, S. Garza y V. Gimeno; Métodos experimentales en la Ingeniería Alimentaria, Editorial Acribia S.A., Zaragoza, España p 263-265 (2000).

Itani, T., M. Tamaki, E. Arai y T. Horino; Distribution of amylose, nitrogen, and minerals in rice kernels with various characters, Journal of Agricultural and Food Chemistry 50, 5326-5332 (2002). 
Lamberts, L., E. De Bie, E. Vandeputte, W. Veraverbeke, V.Derycke, W. De Man y J. Delcour; Effect of milling on colour and nutritional properties of rice, Food Chemistry 100(4): $1496-1503$ (2007).

Lucas, E.; Elaboración de pan. Biotecnología de la fermentación. (2008),

http://www.aldeaeducativa.com/panificaciòn/elaboracióndelpan.html. Acceso: 04 de Abril (2009).

Machado, L.; Pão sem glúten: otimização de algumas variáveis de processamento, Tesis de maestría. Departamento de Ingeniería de Alimentos. Universidade Estadual de Campinas, Campinas, Brasil (1996).

Marco, C. y C. Molina; Mejora de la funcionalidad de proteínas de cereales libres de gluten: Aplicación en productos fermentados, Tesis Doctoral. Universidad de Valencia, Valencia, España (2008).

Mohan, V. y X. Quintero; Propiedades reológicas de alimentos sólidos, En: Rao, M.; S. Rizvi, y A. Datta, Engineering Properties of Foods. $3^{\text {rd }}$ Edition. Taylor \& Francis Group. New York, USA p 99135 (2005)

Montgomery, L.; Diseño y análisis de experimentos, $2^{\text {da }}$ edición. 225-270, Limusa-Wiley, México, (2002).

Muller, H.; Introducción a la Reología de los Alimentos, 144 - 145. Editorial Acribia S.A. Zaragoza, España (1973).

Pedrosa M., C., Airoldi y A. El-Dash; Production of acidic extruded rice flour and its influence on the qualities of gluten-free bread, Food Science and Technology 42(3): 618-623 (2009).

Peleg, M.; Characterization of stress relaxation curves of solid foods, Journal of Food Science. 44 (1): $277-281$ (1979).

Renzetti S. y E. Arendt; Effect of protease treatment on the baking quality of brown rice bread: From textural and rheological properties to biochemistry and microstructure, Journal of Cereal Science 50(1): 22-28 (2009).

Sánchez, H., R. González, C. Osella, R. Torres, y M. de la Torre; Elaboración de pan sin gluten con harinas de arroz extruidas, Revista Ciencia y Tecnología Alimentaria 6(2): 109-116 (2008).

Skinner, G. 1983. Rheological modeling using linear viscoelastic assumptions in static creep and relaxation, Master's thesis, University of Georgia, Georgia (1983).

Stat-Ease Inc.; Software for experiment Design - Expert $^{\circledR}$ versión 6.0.1. Wiley. Minneapolis, USA (2000).

Steffe, J.; Rheological methods in food process engineering, $2^{\text {nd }}$ Edition. 294-310, Freeman Press, Michigan, USA (1996).

Veluppillai, S., K. Nithyanantharajah, S. Vasantharuba, S. Balakumar y V. Arasaratnam; Optimization of Bread Preparation from Wheat Flour and Malted Rice Flour. Rice Science, 17(1): 51-59 (2010). 
